\title{
The importance of Oxalobacter formigenes and oxalic acid in the pathogenesis of chronic kidney disease
}

\author{
Ismail Baloglu' ${ }^{1} \cdot$ Kultigin Turkmen $^{1}$
}

Received: 24 February 2018 / Accepted: 12 March 2018 / Published online: 19 March 2018

๑) Springer Science+Business Media B.V., part of Springer Nature 2018

Editor,

We read with great interest the recent publication 'Gut microbiota and chronic kidney disease: implications for novel mechanistic insights and therapeutic strategies' by Pan et al. [1]. Authors remarked that the intestinal microbiota plays a key role in the pathogenesis of chronic kidney disease (CKD) and CKD-associated complications. In our opinion, some additional data can be added to the pathogenesis of CKD regarding gut and renal axis.

In 1980s oxalic acid was found to be another exogenous cardiotoxic uremic retention molecule that cannot be removed effectively via hemodialysis [2]. Oxalobacter formigenes ( $O$. formigenes) has important roles in oxalate homeostasis by providing both reduction and secretion [3]. Recently, Turkmen et al. [4] hypothesized that serum oxalic acid degradation is impaired mainly due to decreased $O$. formigenes colonization in hemodialysis patients and increased oxalic acid levels might be responsible for increased cardiovascular outcomes in CKD patients. In this regard, our group showed that the colonization of $O$. formigenes was extremely low in HD patients and furthermore we found a positive correlation between serum oxalic acid levels and pulse wave velocity, central aortic systolic/diastolic blood pressures in this population [5]. These changes might be related to the decreased consumption of dietary fiber, delayed colonic transit times, chronic constipation, and impairment of protein assimilation in the small intestine secondary to the uremic milieu in CKD patients [6]. In this context, we also hypothesized that the reduction in serum oxalic acid levels can improve cardiovascular outcomes in HD patients [3].

Ismail Baloglu

i_baloglu@hotmail.com

1 Department of Internal Medicine, Division of Nephrology, Necmettin Erbakan University, Konya, Turkey
Therefore, replacement of $O$. formigenes with probiotics such as natural products might decrease serum oxalic acid levels and improve CKD-associated complications. We hope that the previously mentioned comments might add to the value of the manuscript by Pan et al.

\section{Compliance with ethical standards}

Conflict of interest The authors declare no conflict of interest.

Human and animal rights This article does not contain any unethical studies with human participants or animals performed by any of the authors.

\section{References}

1. Pan Wei, Kang Yongbo (2018) Gut microbiota and chronic kidney disease: implications for novel mechanistic insights and therapeutic strategies. Int Urol Nephrol 50:289-299

2. Mydlik M, Derzsiova K (2010) Oxalic acid—important uremic toxin. Vnitr Lek 56:695-701

3. Kelly JP, Curhan GC, Cave DR, Anderson TE, Kaufman DW (2011) Factors related to colonization with Oxalobacter formigenes in U.S. adults. J Endourol 25:673-679

4. Turkmen K, Erdur FM (2015) The relationship between colonization of Oxalobacter formigenes serum oxalic acid and endothelial dysfunction in hemodialysis patients: from impaired colon to impaired endothelium. Med Hypotheses 84(3):273-275

5. Gulhan B, Turkmen K, Aydin M, Gunay M, Cikman A, Kara M (2015) The relationship between serum oxalic acid, central hemodynamic parameters and colonization by oxalobacter formigenes in hemodialysis patients. Cardiorenal Med 5(3):164-174

6. Kalantar-Zadeh K, Kopple JD, Deepak S, Block D, Block G (2002) Food intake characteristics of hemodialysis patients as obtained by food frequency questionnaire. J Ren Nutr 12:17-31 